\title{
Clinical microbiologists, could we forget the history?
}

\author{
Roberta Migliavacca, ${ }^{1}$ Vittorio Sambri ${ }^{2,3}$ \\ ${ }^{1}$ Department of Clinical, Surgical, Diagnostic and Pediatric Sciences, Unit of Microbiology and Clinical Microbiology, \\ University of Pavia; ${ }^{2}$ Unit of Microbiology, Greater Romagna Hub Laboratory, Pievesestina di Cesena; ${ }^{3}$ DIMES, \\ University of Bologna, Italy
}

\begin{abstract}
Summary
Clinical Microbiologists - whether they are medical doctors or biologists with postgraduate qualification in microbiology and virology - work in healthcare settings undertaking a wide range of laboratory analysis, monitoring microbial cultures and checking new drugs effects. Managing the laboratories, they ensure that data are recorded accurately, in agreement to the most updated clinical and analytical guidelines.

Records analysis and interpretation are only the final steps of a complex processing work, whose quality depends on the choice of the most appropriate test and tool (and technical expertise), in agreement with the samples processing priorities.

The Microbiology Technician figure represents another vital and crucial player in any Clinical Laboratory. This important professional figure is directly responsible for proper handling of all testing samples, any mishandling being able to end in poor testing and consequent inaccurate results.

Microbiology Technicians are involved in setting up all the daily needed laboratory tests for microbial examination and, even before, they have to recognize if the specimens are well suitable for analytical processing. As well, sometimes it is required for these professional personnel to know how properly to store sam-
\end{abstract}

Correspondence: Roberta Migliavacca, Department of Clinical, Surgical, Diagnostic and Pediatric Sciences, Unit of Microbiology and Clinical Microbiology, University of Pavia, Via Brambilla 74, 27100 Pavia, Italy.

Tel.: +39.0382.984143 - Fax: +39.0382 .5484255$

E-mail: r.miglia@unipv.it

Key words: Clinical Microbiologist; automation versus historical bases.

Contributions: RM wrote the manuscript, VS revised the paper.

Conflict of interest: the authors declare no potential conflict of interest.

Received for publication: 29 September 2017.

Accepted for publication: 29 September 2017.

(C) Copyright R. Migliavacca and V. Sambri, 2017

Licensee PAGEPress, Italy

Microbiologia Medica 2017; 32:7108

doi:10.4081/mm.2017.7108

This article is distributed under the terms of the Creative Commons Attribution Noncommercial License (by-nc 4.0) which permits any noncommercial use, distribution, and reproduction in any medium, provided the original author(s) and source are credited. ples and/or strains, for further (confirmatory, deeper, molecular and/or epidemiological) investigations. Most of the microbiological identification work is performed using high-tech equipment, and today's Technicians must be proficient in computer programs to track the complete diagnostic pathway that specimens are following and to find related orders for testing (2).

Different unsolved questions are however emerging, in the present era of microarray-based multiplexing and nucleic-acid-based deep-sequencing methods, which allow simultaneous detection of pathogen nucleic acid and multiple antibiotic resistance (4).

As an example, does the acquisition of the most advanced molecular tools always positively affect the quality of the results and the laboratory productivity?

Could the availability of high throughput instrumentation overcome a wasting time pre-analytical phase?

In which cases do a molecular tool represent a priority? It is well known that MALDI-TOF mass spectrometry allowed us to achieve a more rapid and accurate germ identification results, making costs and work time at the bench lighter (3). Consequently, coupling to MALDI-TOF MS a mecA PCR targeting, positive blood cultures can now be reported up to 1.4 days earlier than it was previously possible, significantly improving the prognosis of MRSA caused bacteraemia by the administration of anti-MRSA active drugs within 48 hours after positive sample detection.

An accurate and rapid diagnosis of infectious diseases is essential for appropriate antimicrobial use, but when will we be ready to discard in vitro bacterial growing tests?

Despite the availability of molecular systems detecting antimicrobial resistance determinants (e.g. Extended Spectrum $\beta$-lactamases and/or carbapenemases genes), we cannot yet achieve, extrapolate MICs values or obtain complex therapy-oriented information, apart from grow-based antimicrobial susceptibility test results. In effect, the presence of a resistance gene does not necessarily lead to treatment failure, because the level of expression may be too low. For example, $\beta$-lactamase production among members of the Enterobacteriaceae is common, but the development of resistance is dependent on the mode and level of expression. Molecular testing could however be required not only for therapy but also to monitor the spread of resistant organisms or resistance genes throughout the hospital and community (1).

Only thanks to a close collaborative interaction between the clinical microbiology laboratory staff (answering potential) and the infectious disease specialists (local epidemiology, administration skills) and clinicians (specific questions), it will be possible to guarantee in the next future results and reports more perfectly fitting with the needs of a single patient management.

Laboratory managers know that essential items to optimize the laboratory performance are: 
i. twenty-four hours availability 365 days a year of a microbiology laboratory

ii. specimens appropriateness for accurate testing (and reporting)

iii. a rapid turnaround time in providing to physicians accurate results

iv. useful information for treatment.

We only have to survive to the risk that an excessive automation may deprive the new generation of microbiology staff of its historical background, which anyway represents the only resource in case of an automated instrument malfunction.

Only keeping alive the historical microbiology bases and promoting the human initiative (e.g. by update lectures on monitoring antibiotic resistance trends, promoting active and passive surveillance programs, and aiming inpatient outbreaks monitoring and control), the jump into the future will be without any precious loss.

\section{References}

1. Fluit AC, Visser MR, Schmitz FJ. Molecular detection of antimicrobial resistance. Clin Microbiol Rev 2001;4:836-71.

2. Peterson LR, Hamilton JD, Baron EJ, et al. Role of clinical microbiology laboratories in the management and control of infectious diseases and the delivery of health care. Clin Infect Dis 2001;32:605-11.

3. Seng P, Abat C, Rolain JM, et al. Identification of rare pathogenic bacteria in a clinical microbiology laboratory: impact of matrix-assisted laser desorption ionization-time of flight mass spectrometry. J Clin Microbiol 2013;51:2182-94.

4. Zumla A, Al-Tawfiq JA, Enne VI, et al. Rapid point of care diagnostic tests for viral and bacterial respiratory tract infections-needs, advances, and future prospects. Lancet Infect Dis 2014;14:1123-35. 